\title{
Exhaled breath condensate in intubated neonates- a window into the lung's glutathione status
}

Maria I Rosso', Susan Roark², Esther Taylor², XiaoDu Ping ${ }^{1}$, Janine M Ward', Katherine Roche², Courtney McCracken ${ }^{1}$, Lou Ann S Brown ${ }^{1,3}$ and Theresa W Gauthier ${ }^{1,3^{*}}$

\begin{abstract}
Background: Analysis of exhaled breath condensates (EBC) is a non-invasive technique to evaluate biomarkers such as antioxidants in the pediatric population, but limited data exists of its use in intubated patients, particularly newborns. Currently, tracheal aspirate (TA) serves as the gold standard collection modality in critically ill newborns, but this method remains invasive. We tested the hypothesis that glutathione status would positively correlate between EBC and TA collections in intubated newborns in the Newborn Intensive Care Unit (NICU). We also hypothesized that these measurements would be associated with alveolar macrophage (AM) glutathione status in the newborn lung.

Methods: Reduced glutathione (rGSH), glutathione disulfide (GSSG), and total GSH (rGSH + (2 X GSSG)) were measured in sequential EBC and TA samples from 26 intubated newborns via high performance liquid chromatography (HPLC). Additionally, AM glutathione was evaluated via immunofluorescence. Pearson's correlation coefficient and associated 95\% confidence intervals were used to quantify the associations between raw and urea-corrected concentrations in EBC and TA samples and AM staining. Statistical significance was defined as $p \leq 0.05$ using two-tailed tests. The sample size was projected to allow for a correlation coefficient of 0.5 , with 0.8 power and alpha of 0.05 .

Results: EBC was obtainable from intubated newborns without adverse clinical events. EBC samples demonstrated moderate to strong positive correlations with TA samples in terms of rGSH, GSSG and total GSH. Positive correlations between the two sampling sites were observed in both raw and urea-corrected concentrations of rGSH, GSSG and total GSH. AM glutathione staining moderately correlated with GSSG and total GSH status in both the TA and EBC.

Conclusions: GSH status in EBC samples of intubated newborns significantly correlated with the GSH status of the TA sample and was reflective of cellular GSH status in this cohort of neonatal patients. Non-invasive EBC sampling of intubated newborns holds promise for monitoring antioxidant status such as GSH in the premature lung. Further studies are necessary to evaluate the potential relationships between EBC biomarkers in the intubated premature newborn and respiratory morbidities.
\end{abstract}

Keywords: Tracheal aspirate, Exhaled breath condensate, Prematurity, Glutathione

\footnotetext{
* Correspondence: tgauthi@emory.edu

'Department of Pediatrics, Emory University School of Medicine, Atlanta, GA, USA

${ }^{3}$ Children's Center for Developmental Lung Biology, Children's Healthcare of

Atlanta, Atlanta, GA, USA

Full list of author information is available at the end of the article
} 


\section{Background}

Bronchopulmonary dysplasia (BPD) is a common complication of survivors of extreme prematurity, causing significant morbidity and mortality among critically ill NICU patients $[1,2]$. The precise pathophysiology of lung injury remains under investigation but it remains clear that inflammation and oxidant stress amplify injury to the premature lung [3-5]. Antioxidant defenses such as glutathione (GSH) are decreased in the premature newborn lung, placing them at increased risk for cellular injury from increased production of reactive oxygen species [6]. Low levels of GSH in the plasma and the bronchoalveolar fluid (BAL) of premature newborns have been implicated in the development of BPD $[7,8]$, and the availability of GSH modulates critical cellular functioning in the lung [9].

Current measurements of oxidant stress and inflammation include evaluation of samples obtained from airway lavage, with tracheal aspirate (TA) sampling being the primary site for the premature newborn $[10,11]$. However, this standard collection modality remains invasive for routine use in newborns, particularly those born prematurely. In recent years, analysis of the volatile and non-volatile constituents in exhaled breath condensate $(\mathrm{EBC})$ has evolved as a novel means to non-invasively study oxidant markers and inflammatory mediators in the airway surface liquid [12-17]. Our laboratory has successfully used this method in previous studies to investigate oxidant stress markers in both asthmatic children [18] and adults [19].

Although TA samples are the mainstay of evaluating samples obtained from premature newborns, there is a lack of data regarding the correlation of the antioxidant status between the TA sample and a non-invasive EBC sample in neonates. We hypothesized that measurements of GSH status obtained via TA collection and EBC collection would positively correlate. Furthermore, since we and others have demonstrated that AM GSH status is dependent on the external milieu of GSH $[9,20]$, we also hypothesized that the GSH status of the EBC and TA would be reflective of cellular GSH in isolated AM. Therefore the goal of this study was to compare the GSH status of a non-invasive EBC sample to its corresponding TA and AM sample to determine whether EBC was reflective of GSH within the neonatal lung and AM.

\section{Methods}

\section{Subjects}

Intubated newborns were enrolled from the NICU at Children's Healthcare of Atlanta at Egleston (CHOA), a level IV intensive care unit within the Emory Division of Neonatology System, in the city of Atlanta from January 2011 through October 2012. Informed consent was obtained from guardians using procedures approved by the Institutional Review Board at Emory University School of Medicine (Gauthier, IRB00038093). All intubated babies were eligible for enrollment. Exclusion criteria included patients deemed too unstable by the attending neonatologist or those on high frequency ventilation, inhaled nitric oxide, or extracorporeal membrane oxygenation (ECMO). Clinical information was ascertained from the electronic medical record.

\section{Exhaled breath condensate (EBC) collection}

Liquid condensate was collected from the exhaled breath of mechanically ventilated newborns for 30 minutes. The EBC collection device consisted of an RTUBE $^{\mathrm{TM}}$ (Respiratory Research, Inc. Austin, TX) encased in an aluminum cooling sleeve that was cooled to $-80^{\circ} \mathrm{C}$ for at least 2 hours prior to use. A modified collection device was used to collect EBC off the ventilator circuit. The RTUBE ${ }^{\mathrm{TM}}$ and cooling sleeve, covered by a blue insulating cover, were connected to the ETT and to the expiratory limb of the ventilator circuit via valve-less connectors provided in most standard ventilator circuits (Figure 1A). Figure 1B depicts a simulated collection from an intubated neonatal mannequin. Immediately after collection, the condensate tubes were capped, placed on wet ice and transported to the laboratory for analyses. The process of EBC collection was monitored at the bedside by the investigators (MR, SR, ET) and was tolerated well by all subjects, without significant changes in heart rate, oxygen saturation or blood pressure.

\section{Tracheal aspirate (TA) collection}

TA samples where obtained within one hour of EBC collection. For the suctioning procedure, bacteriostatic saline $(\sim 1 \mathrm{ml})$ was instilled into the trachea. After several ventilator breaths, an in-line suction catheter (Ballard, Kimberly-Clark, Irving, TX) was inserted just past the endotracheal tube and the sample was then retrieved into a closed, sterile Lukens trap (Covidien, Mansfield, MA). Additional bacteriostatic saline was used if necessary to clear the sample from the suction catheter. Samples were immediately placed on wet ice and transported to the laboratory for analyses.

\section{Sample processing and high performance liquid chromatography (HPLC) analysis}

Patients and samples were de-identified with a study number to ensure confidentiality. All samples were transported to the laboratory on wet ice within an hour of collection. In previous studies, we determined that the rate of oxidation was $\sim 5 \%$ per hour if the sample was maintained in a sterile Lukens trap on wet ice [21]. TA samples were centrifuged for 10 minutes to remove the cell pellet. EBC was extracted from the RTUBE with a plunger apparatus. To prevent auto-oxidation before analysis, $240 \mu \mathrm{L}$ of the EBC was aliquoted into pre-made tubes containing $21 \mu \mathrm{L}$ of a diluted preservative with a final concentration of $5 \%$ perchloric 


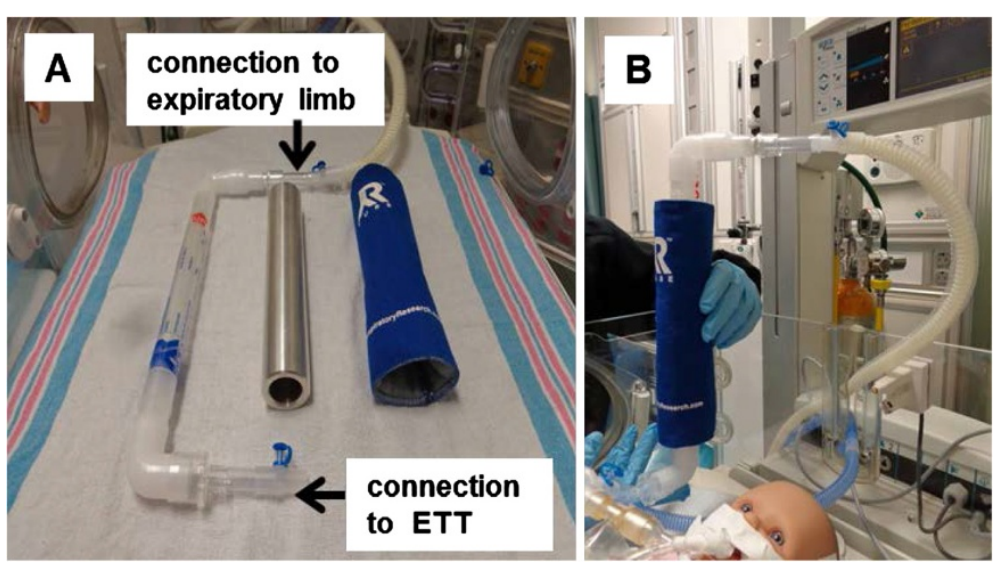

Figure $1 \mathrm{EBC}$ collection from the expiratory limb of the ventilator. A) The RTUBE ${ }^{\mathrm{TM}}$ and its cooling sleeve covered by the blue insulated cover where connected to the expiratory limb of the ventilator circuit via valve-less connectors. B) A simulated collection of EBC on an intubated neonatal mannequin.

acid, $0.1 \mathrm{M}$ boric acid, $6.7 \mathrm{mM}$ iodoacetic acid (IAA), and 5 $\mathrm{nM}$ of the internal standard $\gamma$-glutamyl-glutamate as we have previously described [22]. For TA, $250 \mu \mathrm{L}$ of sample was added to pre-made tubes containing $250 \mu \mathrm{L}$ of preservative for a final concentration of $10 \%$ perchloric acid, $0.2 \mathrm{M}$ boric acid, 13.4 mM IAA, and $10 \mu \mathrm{M} \gamma$-glutamylglutamate. All samples were stored at $-80^{\circ} \mathrm{C}$ until batch analysis by HPLC, also previously described by this laboratory $[21,23]$. GSH and GSSG fractions were treated with dansyl chloride and the dansylated derivatives separated using an amino $\mu$ BondaPak column (Waters, Milford MA) with fluorescent detection. The lower limits of detection were $15 \mathrm{fmol} / \mu \mathrm{l}$ for $\mathrm{rGSH}$ and were consistent with studies by Komatsu and Obata [24] which used similar methods and reported similar lower limits of detection. All of the samples in this study were above the noted limits of detection. To minimize operational variability, all samples from this study were analyzed within the same HPLC run. Inter-assay coefficient of variability was determined by performing HPLC analysis on a mixture of standards including cysteine, cystine, rGSH, and GSSG. This mixture was analyzed immediately before, in the middle and at the end of sample set analysis. Based on this cocktail of standards, the inter-assay coefficient of variability was calculated at $1 \%$. Given the limited sample volume obtained, we were unable to calculate intra-assay variability.

The subject's blood urea nitrogen level (BUN, mg/dl) was noted in the medical record on the day samples were obtained. Sample urea was measured using the Bethleot/ Colorimetric assay (Pointe Scientific, Canton, MI - sensitivity range of 0.05 to $150 \mathrm{mg} / \mathrm{dl})$. Sample concentrations of rGSH, GSSG and total GSH (defined as: GSH + (2 X GSSG)) are presented as both raw values and urea corrected values. Samples were corrected for dilution by multiplication with the urea dilution factor (f) defined as $\mathrm{f}=$ Patient BUN/ sample urea used was as previously described for EBC samples [21].

\section{AM immunofluorescence}

TA samples where centrifuged as noted above to obtain cells (300 g, 10 minutes). The supernatant was processed as above and the cell pellets where re-suspended in RPMI 1640 media with 10\% fetal calf serum (FCS) and plated under sterile conditions for 1 hour. The media was removed and the adhered cells washed three times with phosphate buffered saline (PBS). Cells where then fixed with $4 \%$ paraformaldehyde solution for 30 minutes, washed with PBS and stored at $4^{\circ} \mathrm{C}$. At the time of analysis non-specific binding was blocked with $10 \%$ bovine serum albumin (BSA, 1 hour) and the cells were stained for protein-glutathione adducts (primary mouse monoclonal IgG GSH, 2 hours) (Virogen, Watertown, MA). After washing, a secondary Alexa fluorescence 488 green anti-mouse IgG was added for 2 hours (Life Technologies, Carlsbad, CA). The cells were identified as AM by cell morphology and then analyzed for fluorescence via confocal fluorescent microscopy (Olympus FluoView FV1000). The cellular fluorescence was quantified as relative fluorescent units (RFU) /cell as we previously described [25].

\section{Statistical analyses}

All data were analyzed with $\mathrm{SPSS}^{\odot}$ for Windows software (Version 18.0, SPSS Inc., Chicago, IL) and SAS 9.3 (Cary, NC). Prior to analysis, data were assessed for normality using histograms, normal probability plots, and the Kolmogorov-Smirnov test of normality. For concentrations that were non-normally distributed, a $\log _{10}$ normalizing transformation was applied. Pearson's correlation coefficient and associated 95\% confidence intervals were 
used to quantify the association between TA and EBC concentrations. Scatter plots were used to assess whether the relationship between two concentrations appeared linear and to confirm that the Pearson's correlation coefficient was an appropriate measure of association. Statistical significance was defined as $\mathrm{p} \leq 0.05$ using two-tailed tests. The sample size was determined in order to detect a minimum correlation coefficient of 0.5 , with 0.8 power and an alpha of 0.05 .

\section{Results}

\section{Study population}

Twenty-six (26) intubated newborns on mechanical ventilation were enrolled (Table 1). Enrolled subjects had a median birthweight of 1564 grams (interquartile range (IQR) 698, 2914 grams) and a median gestational age of 32.5 weeks (IQR: 24, 38 weeks). At the time of EBC and TA collection, the median weight was 2280 grams (IQR: 1500,3254 grams) with a corrected gestational age of 35 weeks (IQR: 34, 39.5 weeks). Subjects were ventilated for a mean of 21 days (IQR: 7, 59.5 days) at the time of sample collection. As noted in Table 1, the majority of patients were admitted for surgical procedures.

GSH status was measureable in both TA and EBC samples The median volume recovered from the TA was $0.75 \mathrm{ml}$ (IQR: 0.6, $1.75 \mathrm{ml}$ ) while the median EBC volume recovered after 30 minutes of sampling was $2.2 \mathrm{ml}$ (IQR: 1.5, $2.5 \mathrm{ml})$. GSH, GSSG and total GSH were detected by HPLC in all samples (100\%) in the nM range. The urea dilution factors, raw concentrations, and urea-corrected concentrations are presented in Table 2.

\section{TA and EBC concentrations of rGSH and GSSG significantly correlated}

We postulated that rGSH and GSSG values measured in the TA would correlate with those measured via a noninvasive EBC. As seen in Figure 2, urea-corrected rGSH in the TA demonstrated a moderate positive correlation with urea-corrected rGSH in the EBC. Similarly, concentrations of TA rGSH not normalized to urea moderately correlated with EBC rGSH concentrations not normalized to urea ( $r=0.574, \mathrm{p}=0.002,95 \% \mathrm{CI}:(0.230-0.782)$, $\mathrm{R}^{2}=0.329$ ). Interestingly, the duration of mechanical ventilation demonstrated a moderate negative correlation with urea-corrected rGSH in the EBC $(r=-0.574, \mathrm{p}=$ 0.003 , 95\% CI: $\left(-0.779-(-0.209), \mathrm{R}^{2}=0.329\right)$ but did not correlate with urea-corrected rGSH in the TA $(\mathrm{p}=\mathrm{NS})$. Figure 3 demonstrates a strong positive correlation between urea-corrected GSSG in the TA and urea-corrected GSSG in the EBC. A similarly strong positive correlation existed between TA GSSG concentrations not normalized by urea and EBC GSSG concentrations not normalized to urea $\left(\mathrm{r}=0.708, \mathrm{p}<0.001,95 \%\right.$ CI: $(0.431-0.856), \mathrm{R}^{2}=$
0.501). Although the fraction of oxidized GSH (i.e. the \% GSSG) was approximately two times higher in the EBC than in the TA (EBC: $44.8 \pm 4.6 \%$ vs TA: $21.8 \pm 3 \%$, p < 0.001 ), there was no significant correlation between the TA \% GSSG and the EBC \% GSSG ( $\mathrm{p}=\mathrm{NS})$. When total GSH was compared between the two sampling sites, a moderate positive correlation was demonstrated in both urea-corrected values (Figure 4) and in non-urea normalized values $(r=0.649, \mathrm{p}<0.001,95 \% \mathrm{CI}:(0.338-0.824)$, $\left.\mathrm{R}^{2}=0.421\right)$.

\section{GSH status in both TA and EBC correlated with AM GSH staining}

Since we hypothesized that the GSH in the TA or EBC sample may be reflective of cellular GSH in the isolated $\mathrm{AM}$, we evaluated the correlation of total GSH measured in TA and EBC with AM GSH staining determined via immunofluorescence. Fluorescent staining for protein-GSH adducts in the AM demonstrated a moderate positive correlation with the urea-corrected concentration of total GSH in the TA and the EBC (Figure 5A and $\mathrm{B})$. Furthermore, AM demonstrated a moderate positive correlation with urea-corrected concentrations of GSSG in the TA (Figure 6A) and in the EBC (Figure 6B). While we did not observe a correlation between AM GSH and urea-corrected concentrations of rGSH in the TA ( $\mathrm{p}=\mathrm{NS}$ ), a moderate positive correlation was found with urea-corrected concentrations of rGSH in the EBC ( $r=$ $0.571, \mathrm{p}=0.004,95 \%$ CI: $\left.(0.195-0.791), \mathrm{R}^{2}=0.326\right)$.

\section{Discussion}

In the current investigation, our main goals were 1) to determine if the status of the antioxidant GSH measured in a TA sample correlated with that of a corresponding EBC sample and 2) to determine whether EBC collection enabled a safe, non-invasive approach to evaluate such data in an intubated neonatal population. No adverse events where noted during EBC collection in our series of ventilated neonatal patients. Both the raw and ureacorrected concentrations of EBC rGSH, GSSG and total GSH significantly correlated with those measurements in the more invasive TA samples. Furthermore, this study demonstrated that both the total GSH and the GSSG in TA and EBC samples positively correlated with the cellular GSH staining of isolated AM.

Collection of EBC is based on the premise that airway surface liquid droplets are entrained and mixed with water vapor and other volatile gases during tidal breathing [17]. Upon exhalation, the breath is cooled in a passive sampling device which causes water vapor to condense into respiratory droplets plus other exhaled particles and collect on the chilled walls of the sampling device. Investigations into the use of EBC as a non-invasive tool to monitor pulmonary biomarkers of disease have gained much 
Table 1 Patient demographics

\begin{tabular}{|c|c|c|c|c|c|c|c|}
\hline Subject number & GA (weeks) & $\begin{array}{l}\text { GA at sample } \\
\text { collection (weeks) }\end{array}$ & Birth weight (grams) & $\begin{array}{l}\text { Weight at sample } \\
\text { collection (grams) }\end{array}$ & $\begin{array}{l}\text { BUN at sample } \\
\text { collection }(\mathrm{mg} / \mathrm{dl})\end{array}$ & $\begin{array}{l}\text { Duration of mechanical } \\
\text { ventilation (days) }\end{array}$ & Primary diagnosis \\
\hline 1 & 37 & 40 & 2442 & 2760 & 3 & 21 & Hypoglossia-hypodactylia syndrome \\
\hline 2 & 24 & 27 & 675 & 1000 & 6 & 21 & Spontaneous intestinal perforation \\
\hline 3 & 26 & 35 & 686 & 1730 & 3 & 189 & Patent ductus arteriosus \\
\hline 4 & 24 & 35 & 690 & 3780 & 8 & 77 & Subglottic stenosis \\
\hline 5 & 35 & 42 & 1980 & 3000 & 12 & 49 & Congenital diaphragmatic hernia \\
\hline 6 & 41 & 42 & 3530 & 4060 & 10 & 7 & Esophageal atresia/tracheal esophageal fistula \\
\hline 7 & 39 & 40 & 3800 & 3850 & 5 & 7 & Neonatal seizures \\
\hline 8 & 37 & 40 & 2782 & 3365 & 7 & 21 & Laryngeal cleft \\
\hline 9 & 26 & 34 & 803 & 1650 & 5 & 56 & Jejunal stricture \\
\hline 10 & 24 & 34 & 720 & 1895 & 1 & 70 & Pneumoperitoneum \\
\hline 11 & 24 & 30 & 760 & 1380 & 18 & 42 & Necrotizing enterocolitis \\
\hline 12 & 38 & 40 & 3100 & 2940 & 19 & 14 & Gastroschisis \\
\hline 13 & 38 & 39 & 2858 & 2858 & 13 & 7 & Gastroschisis \\
\hline 14 & 39 & 40 & 3080 & 3265 & 18 & 7 & Imperforate anus/congenital heart disease \\
\hline 15 & 35 & 36 & 2440 & 2440 & 16 & 7 & Gastroschisis \\
\hline 16 & 24 & 26 & 700 & 810 & 29 & 14 & Spontaneous intestinal perforation \\
\hline 17 & 38 & 38 & 3590 & 3590 & 12 & 5 & Congenital diaphragmatic hernia \\
\hline 18 & 31 & 33 & 1417 & 1100 & 26 & 14 & Congenital heart disease, duodenal atresia \\
\hline 19 & 34 & 34 & 2120 & 2120 & 15 & 4 & Multiple jejunal atresias \\
\hline 20 & 35 & 43 & 1710 & 2500 & 16 & 56 & Intestinal perforation, microcolon \\
\hline 21 & 39 & 41 & 3170 & 3250 & 41 & 14 & Pentalogy of cantrell \\
\hline 22 & 25 & 37 & 450 & 1530 & 33 & 84 & Extreme prematurity \\
\hline 23 & 24 & 32 & 550 & 1200 & 18 & 56 & Extreme prematurity \\
\hline 24 & 23 & 34 & 610 & 1410 & 2 & 77 & Spontaneous intestinal perforation \\
\hline 25 & 27 & 35 & 1110 & 1900 & 3 & 56 & Patent ductus arteriosus \\
\hline 26 & 25 & 35 & 850 & 1650 & 33 & 70 & Jejunal perforation \\
\hline
\end{tabular}


Table 2 rGSH, GSSG and total GSH are measurable in both TA and EBC samples

\begin{tabular}{lcc}
\hline & TA & EBC \\
\hline Collected fluid volume $(\mathbf{m l})$ & $0.75(0.6,1.75)$ & $2.2(1.5,2.5)$ \\
Sample urea $(\mathbf{m g} / \mathbf{d l})$ & $11.2(3.8,28.0)$ & $3.3(3.1,28.3)$ \\
Urea dilution factor & $0.69(0.42,3.77)$ & $0.90(0.49,4.87)$
\end{tabular}

Sample GSH (raw values, nM)

\begin{tabular}{lcc} 
rGSH & $155(71,1087)$ & $0.5(0.4,37)$ \\
GSSG & $112.0(7.9,345)$ & $1.0(0.8,10.9)$ \\
Total GSH & $273(107,1831)$ & $2.3(2.1,63.7)$ \\
$\begin{array}{l}\text { Sample GSH (urea-corrected } \\
\text { values, nM) }\end{array}$ & \\
rGSH & & \\
GSSG & $225(107,562)$ & $3.8(0.5,12.2)$ \\
Total GSH & $45.5(20.9,191)$ & $4.3(1.3,8.6)$ \\
\hline
\end{tabular}

Values are presented as median (IQR)

interest in the last decade [13]. Analysis of EBC has led to novel observations about the antioxidant status of the lung in varying disease states in both the adult and pediatric population [16,26]. Our laboratory has successfully used this method in previous studies to demonstrate diminished GSH availability in the lungs of asthmatic children [18] and adults [19].

Although EBC use has widely been studied with the hopes of utilizing this technique in clinical practice in the adult arena [12], data investigating $\mathrm{EBC}$ in newborn infants remains limited. Evaluation of EBC has been described in spontaneously breathing infants with a median age of approximately 2 years [27] and in older children

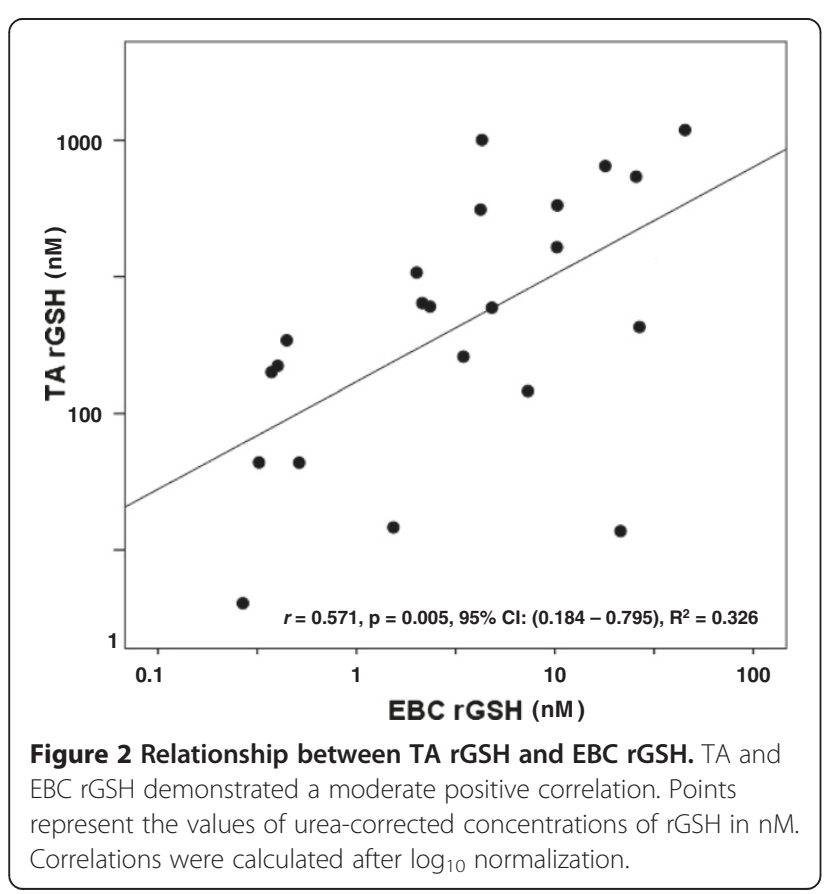

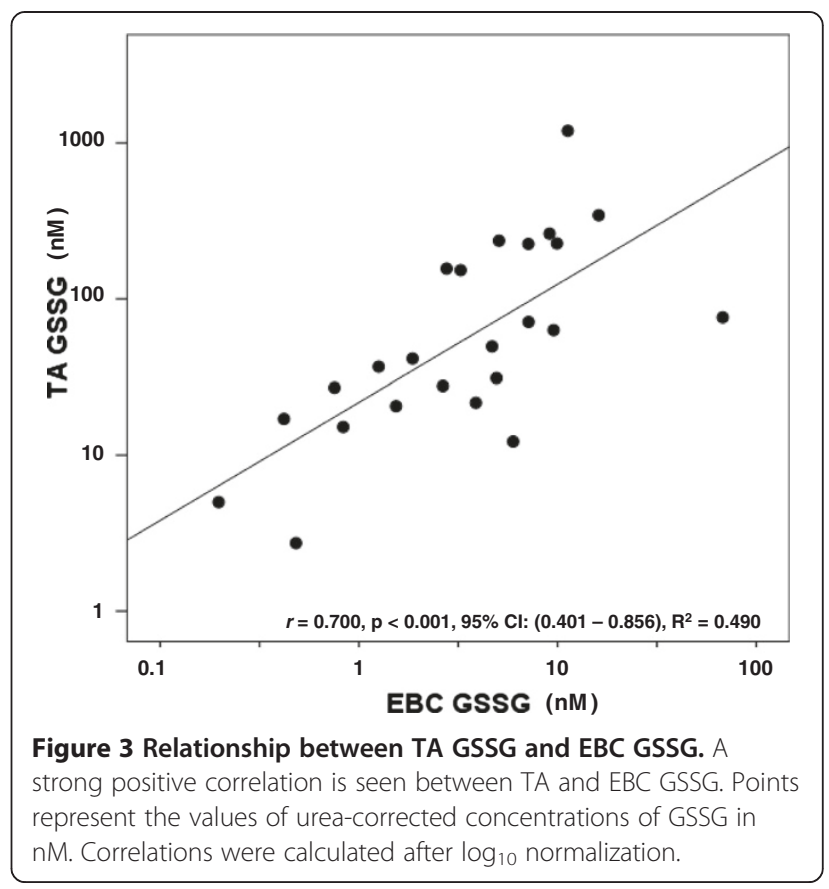

between 1-30 months of age [28]. Collection of EBC via the exhalation limb of the ventilator has been reported in intubated pediatric patients [29] and in neonates requiring mechanical ventilation or CPAP [30]. However, the current study is the first report to our knowledge aimed at comparing TA and EBC samples in an exclusively intubated neonatal population. Our data suggest significant correlations existed between EBC and TA GSH markers in neonates, unlike studies in adult subjects, where $\mathrm{EBC}$ and BAL

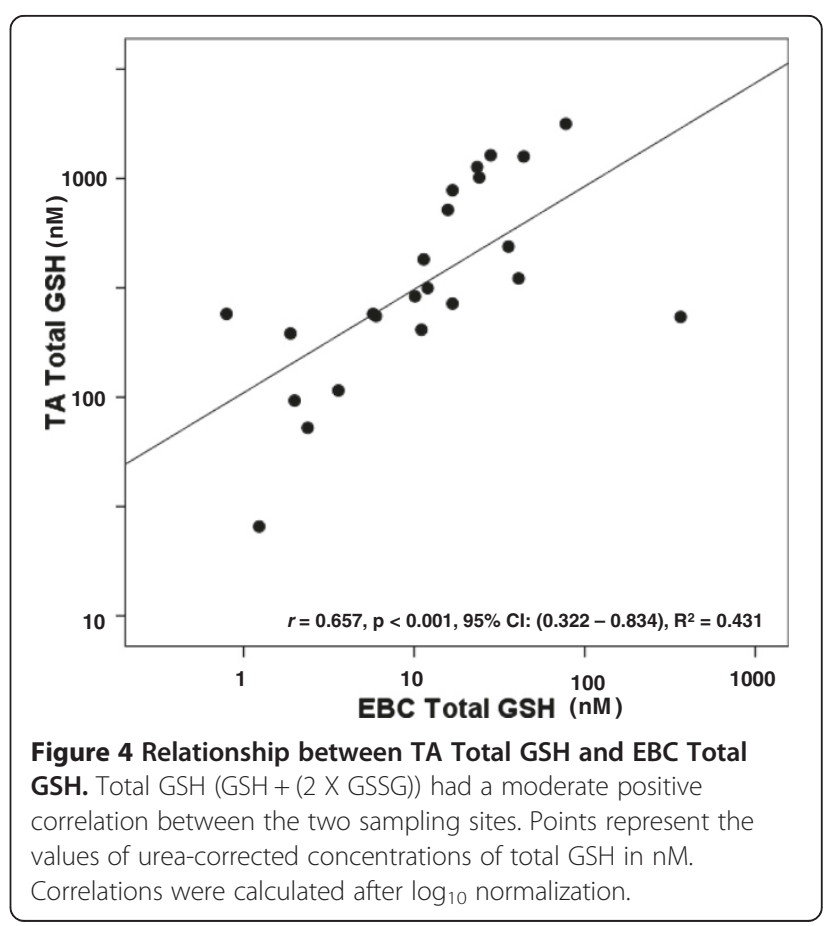



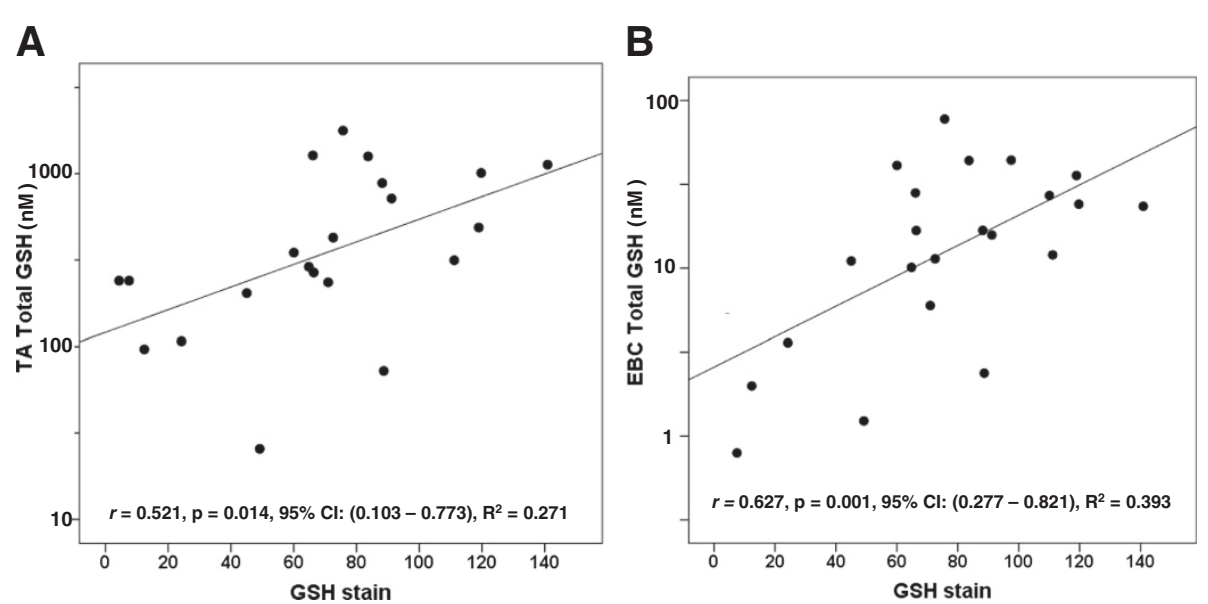

Figure 5 Relationship between TA and EBC total GSH and AM GSH staining. Total GSH concentrations in the A) TA and B) EBC as measured via HPLC were compared to GSH staining on the isolated AM as determined by immunofluorescence. A) A moderate positive correlation existed between TA total GSH and AM GSH stain. B) A moderately positive correlation also exists between EBC total GSH and AM GSH. Points represent the values of urea-corrected total GSH concentrations in $\mathrm{nM}$ on the $\mathrm{Y}$ axis versus AM GSH stain as quantified in relative fluorescent units (RFU)/cell on the $\mathrm{X}$ axis. Correlations were calculated after $\log _{10}$ normalization of the total GSH concentrations.

biomarkers such exhaled nitric oxide, 8-isoprostane and hydrogen peroxide did not correlate [31].

The strengths of the current study include a standardized approach to the collection of the EBC in terms of methodology and timing. EBC was collected from the exhalation limb of the ventilator in intubated newborns which reduced possible contamination from volatiles in the atmosphere or the mouth as has been demonstrated in spontaneously breathing subjects [12-14].

This study has several limitations. The goal of this project was to determine correlations between non-invasive $\mathrm{EBC}$ collection and TA collection; for this reason EBC and TA samples were drawn sequentially from each patient as to compare them to each other and not between population subsets. As such, the sample size of this pilot study was small. A much larger sample size would be required to appropriately control for gestational age, chronological age of the newborn, and respiratory diagnosis. However, data from this pilot study justifies a much larger, adequately powered clinical study aimed to define normative values in such a neonatal population.

During the study we did not monitor temperature stability throughout sample collections. The R-Tubes were precooled to $-80^{\circ} \mathrm{C}$ for a minimum of 2 hours, kept in an insulated glove and placed on wet ice prior to and immediately after sample collection. However, the R tube has
A

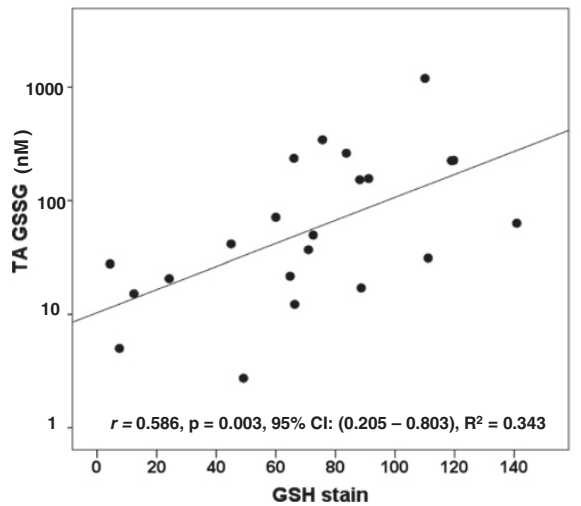

B

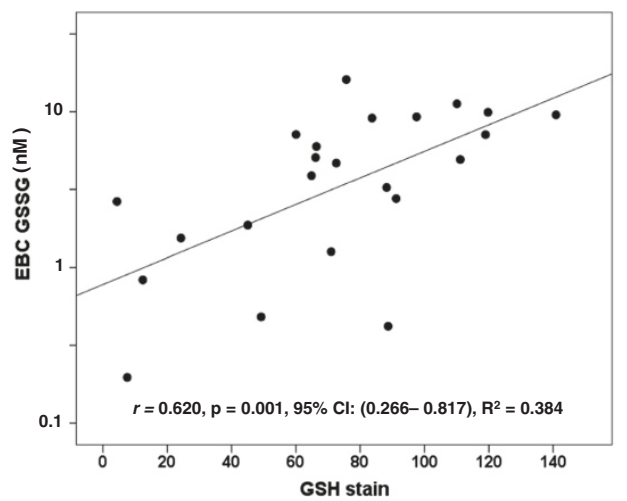

Figure 6 Relationship between TA and EBC GSSG and AM GSH staining. GSSG concentration in the A) TA and B) EBC were compared to GSH staining on the isolated AM. A) A moderate positive correlation existed between GSSG in the TA and AM GSH stain. B) A moderately positive correlation also exists between GSSG in the EBC and AM GSH. Points represent the values of urea-corrected GSSG concentrations in nM on the $\mathrm{Y}$ axis versus AM GSH stain as quantified in relative fluorescent units (RFU)/cell on the $\mathrm{X}$ axis. Correlations were calculated after $\log _{10}$ normalization of the total GSH concentrations. 
been shown to be sensitive to ambient temperatures and varying temperature has been noted to affect $\mathrm{EBC}$ analyses $[13,14,32-34]$.

The standardization of EBC analyses remains limited since normalization for dilution within the EBC samples remains problematic. Current literature outlines an ongoing debate regarding the interpretation of dilution in EBC sampling [17,35-37]. While we attempted to control for dilution using the urea-correction, as is acceptable for BAL analyses, it remains unknown whether this method is applicable to EBC sampling [14]. Although the use of urea-correction for $\mathrm{EBC}$ is not yet established, our data suggest that this may be an appropriate method to address the effects of dilution in both TA and EBC sampling, since similar correlations were noted.

Investigations into the GSH status of premature newborns remain clinically important. Advances in modern neonatal respiratory support and clinical interventions for the prevention or treatment of BPD have not succeeded in reducing its occurrences [2]. Therefore, continued investigations into the antioxidant status of the at-risk premature newborn lung may allow patient-specific therapies to improve respiratory outcomes in this at-risk population [38]. Even though the thiol pair of GSH/GSSG is essential for normal cellular functioning and signaling in the lung [39], there remains limited information regarding its abundance in the premature newborn [8], who is particularly at risk for both inflammatory [40] and oxidative injury [6]. TA sampling, albeit less invasive than a formal BAL, still requires invasive suctioning of the airway. EBC holds potential as a non-invasive approach to evaluating GSH status in the neonatal lung, and to assist in identifying those particular infants at greatest risk for the development of BPD. The current study demonstrates feasibility of this approach in an intubated neonatal population. Interestingly, a moderate negative correlation was demonstrated between EBC rGSH and the duration of mechanical ventilation at the time of sample collection. Furthermore, since GSH availability modulates functioning of the resident AM $[9,25]$, determining the status of GSH availability in the developing lung could advance our understanding of the mechanisms underlying antioxidant depletion and the development of BPD in the neonate.

\section{Conclusion}

EBC collection in intubated neonates was well tolerated without significant changes in respiratory status. GSH status in the EBC positively correlated with that obtained in the more invasive TA, and positively correlated with that seen in isolated AM. Evaluation of EBC holds promise to monitor antioxidant status, such as GSH in premature newborns. Further investigations are required to incorporate this technology to assist in identifying those infants at greatest risk for subsequent oxidant-induced injury in the lung.

\section{Abbreviations}

EBC: Exhaled breath condensates; TA: Tracheal aspirates; rGSH: Reduced glutathione, GSSG, glutathione disulfide; AM: Alveolar macrophage; NICU: Newborn intensive care unit; HPLC: High performance liquid chromatography; BPD: Bronchopulmonary dysplasia; BAL: Bronchoalveolar fluid; ECMO: Extracorporeal membrane oxygenation; IAA: lodoacetic acid; BUN: Blood urea nitrogen level; IQR: Interquartile range.

\section{Competing interests}

The authors have no financial ties or conflicts of interest to disclose.

\section{Authors' contributions}

Study idea and design: MR, SR, ET, LAB, TG; Patient enrollment and sample collection: MR, SR, ET, KR, TG; Sample processing and analyses: MR, XP, JW $\mathrm{KR}$; Statistical analyses and interpretation of data: MR, CM, LAB, TG. Manuscript drafting and revision: MR, CM, LAB, TG. All authors read and approved the final manuscript.

\section{Author details}

'Department of Pediatrics, Emory University School of Medicine, Atlanta, GA USA. ${ }^{2}$ Children's Healthcare of Atlanta, Atlanta, GA, USA. ${ }^{3}$ Children's Center for Developmental Lung Biology, Children's Healthcare of Atlanta, Atlanta, GA USA

Received: 29 August 2013 Accepted: 2 January 2014 Published: 7 January 2014

References

1. Jobe AH: The new bronchopulmonary dysplasia. Curr Opin Pediatr 2011, 23(2):167-172.

2. Stoll BJ, Hansen NI, Bell EF, Shankaran S, Laptook AR, Walsh MC, Hale EC, Newman NS, Schibler K, Carlo WA, et al: Neonatal outcomes of extremely preterm infants from the NICHD neonatal research network. Pediatrics 2010, 126(3):443-456.

3. Viscardi RM: Perinatal inflammation and lung injury. Semin Fetal Neonatal Med 2012, 17(1):30-35

4. Bustani $P$, Kotecha S: Role of cytokines in hyperoxia mediated inflammation in the developing lung. Front Biosci 2003, 8:5694-704.

5. Jobe $\mathrm{AH}$, Ikegami M: Prevention of bronchopulmonary dysplasia. Curr Opin Pediatr 2001, 13(2):124-129.

6. Davis JM, Auten RL: Maturation of the antioxidant system and the effects on preterm birth. Semin Fetal Neonatal Med 2010, 15(4):191-195.

7. Jain A, Mehta T, Auld PA, Rodrigues J, Ward RF, Schwartz MK, Martensson J: Glutathione metabolism in newborns: evidence for glutathione deficiency in plasma, bronchoalveolar lavage fluid, and lymphocytes in prematures. Pediatr Pulmonol 1995, 20(3):160-166.

8. Grigg J, Barber A, Silverman M: Bronchoalveolar lavage fluid glutathione in intubated premature infants. Arch Dis Child 1993, 69(1 Spec No):49-51.

9. Gauthier TW, Ping XD, Harris FL, Wong M, Elbahesh H, Brown LA: Fetal alcohol exposure impairs alveolar macrophage function via decreased glutathione availability. Pediatr Res 2005, 57(1):76-81.

10. Harwood DT, Darlow BA, Cheah FC, McNeill N, Graham P, Winterbourn CC Biomarkers of neutrophil-mediated glutathione and protein oxidation in tracheal aspirates from preterm infants: association with bacterial infection. Pediatr Res 2011, 69(1):28-33.

11. Schneibel KR, Fitzpatrick AM, Ping XD, Brown LA, Gauthier TW: Inflammatory mediator patterns in tracheal aspirate and thei association with bronchopulmonary dysplasia in very low birth weight neonates. J Perinatol 2013, 35(5):383-387.

12. Horvath I, Hunt J, Barnes PJ, Alving K, Antczak A, Baraldi E, Becher G, van Beurden WJ, Corradi M, Dekhuijzen R, et al: Exhaled breath condensate: methodological recommendations and unresolved questions. Eur Respir $J$ 2005, 26(3):523-548

13. Ahmadzai $H$, Huang S, Hettiarachchi R, Lin JL, Thomas PS, Zhang Q: Exhaled breath condensate: a comprehensive update. Clin Chem Lab Med 2013, 51(7):1343-1361

14. Rosias P: Methodological aspects of exhaled breath condensate collection and analysis. J Breath Res 2012, 6(2):027102.

15. Hunt J: Exhaled breath condensate $\mathrm{pH}$ : reflecting acidification of the airway at all levels. Am J Respir Crit Care Med 2006, 173(4):366-367. 
16. Greenwald R, Fitzpatrick AM, Gaston B, Marozkina NV, Erzurum S, Teague WG: Breath formate is a marker of airway S-nitrosothiol depletion in severe asthma. PLOS ONE 2010, 5(7):e11919.

17. Liang Y, Yeligar SM, Brown LA: Exhaled breath condensate: a promising source for biomarkers of lung disease. Scientific World J 2012, 2012:217518.

18. Fitzpatrick AM, Teague WG, Holguin F, Yeh M, Brown LA: Airway glutathione homeostasis is altered in children with severe asthma: evidence for oxidant stress. J Allergy Clin Immunol 2009, 123(1):146-152. e148.

19. Yeh MY, Burnham EL, Moss M, Brown LA: Non-invasive evaluation of pulmonary glutathione in the exhaled breath condensate of otherwise healthy alcoholics. Respir Med 2008, 102(2):248-255.

20. Forman HJ, Skelton DC: Protection of alveolar macrophages from hyperoxia by gamma-glutamyl transpeptidase. Am J Physiol 1990, 259(2 Pt 1):L102-107.

21. Yeh MY, Burnham EL, Moss M, Brown LA: Chronic alcoholism alters systemic and pulmonary glutathione redox status. Am J Respir Crit Care Med 2007, 176(3):270-276.

22. Fitzpatrick AM, Teague WG, Burwell L, Brown MS, Brown LA: Glutathione oxidation is associated with airway macrophage functional impairment in children with severe asthma. Pediatr Res 2011, 69(2):154-159.

23. Gauthier TW, Kable JA, Burwell L, Coles CD, Brown LA: Maternal alcohol use during pregnancy causes systemic oxidation of the glutathione redox system. Alcohol Clin Exp Res 2010, 34(1):123-130.

24. Komatsu H, Hoshino A, Funayama M, Kawahara K, Obata F: Oxidative modulation of the glutathione-redox couple enhances lipopolysaccharide-induced interleukin 12 P40 production by a mouse macrophage cell line, J774A.1. Free Radic Res 2003, 37(3):293-299.

25. Gauthier TW, Ping XD, Gabelaia L, Brown LA: Delayed neonatal lung macrophage differentiation in a mouse model of in utero ethanol exposure. Am J Physiol Lung Cell Mol Physiol 2010, 299(1):L8-16.

26. Rahman I, Biswas SK: Non-invasive biomarkers of oxidative stress: reproducibility and methodological issues. Redox Rep 2004, 9(3):125-143.

27. Vogelberg C, Wurfel C, Knoetzsch A, Kahlert A, Range U, Leupold W: Exhaled breath condensate $\mathrm{pH}$ in infants and children with acute and recurrent wheezy bronchitis. Pediatr Pulmonol 2007, 42(12):1166-1172.

28. Moeller A, Franklin P, Hall GL, Horak F Jr, Wildhaber JH, Stick SM: Measuring exhaled breath condensates in infants. Pediatr Pulmonol 2006, 41(2):184-187.

29. Walsh BK, Mackey DJ, Pajewski T, Yu Y, Gaston BM, Hunt JF: Exhaled-breath condensate $\mathrm{pH}$ can be safely and continuously monitored in mechanically ventilated patients. Respir Care 2006, 51(10):1125-1131.

30. Cheah FC, Darlow BA, Winterbourn CC: Problems associated with collecting breath condensate for the measurement of exhaled hydrogen peroxide from neonates on respiratory support. Biol Neonate 2003, 84(4):338-341.

31. Jackson AS, Sandrini A, Campbell C, Chow S, Thomas PS, Yates DH Comparison of biomarkers in exhaled breath condensate and bronchoalveolar lavage. Am J Respir Crit Care Med 2007, 175(3):222-227.

32. Folesani G, Corradi M, Goldoni M, Manini P, Acampa O, Andreoli R, Bertorelli G, David S, Franchini l: Urea in exhaled breath condensate of uraemics and patients with chronic airway diseases. Acta Biomed 2008, 79(1):79-86

33. Knobloch $\mathrm{H}$, Becher $\mathrm{G}$, Decker $\mathrm{M}$, Reinhold P: Evaluation of $\mathrm{H} 2 \mathrm{O} 2$ and $\mathrm{pH}$ in exhaled breath condensate samples: methodical and physiological aspects. Biomarkers 2008, 13(3):319-341.

34. Czebe K, Barta I, Antus B, Valyon M, Horvath I, Kullmann T: Influence of condensing equipment and temperature on exhaled breath condensate $\mathrm{pH}$, total protein and leukotriene concentrations. Respir Med 2008, 102(5):720-725.

35. Esther CR Jr, Boysen G, Olsen BM, Collins LB, Ghio AJ, Swenberg JW, Boucher RC: Mass spectrometric analysis of biomarkers and dilution markers in exhaled breath condensate reveals elevated purines in asthma and cystic fibrosis. Am J Physiol Lung Cell Mol Physiol 2009, 296(6):L987-993.

36. Effros RM: Exhaled breath condensate: delusion or dilution? Chest 2010, 138(3):471-472

37. Kazani S, Israel E: Exhaled breath condensates in asthma: diagnostic and therapeutic implications. J Breath Res 2010, 4(4):047001.
38. Welty SE: Is there a role for antioxidant therapy in bronchopulmonary dysplasia? J Nutr 2001, 131(3):947S-950S.

39. Jones DP: Redefining oxidative stress. Antioxid Redox Signal 2006 8(9-10):1865-1879.

40. Wright $\mathrm{CJ}$, Kirpalani $\mathrm{H}$ : Targeting inflammation to prevent bronchopulmonary dysplasia: can new insights be translated into therapies? Pediatrics 2011, 128(1):111-126.

doi:10.1186/1465-9921-15-1

Cite this article as: Rosso et al:: Exhaled breath condensate in intubated neonates- a window into the lung's glutathione status. Respiratory Research 2014 15:1.

\section{Submit your next manuscript to BioMed Central and take full advantage of:}

- Convenient online submission

- Thorough peer review

- No space constraints or color figure charges

- Immediate publication on acceptance

- Inclusion in PubMed, CAS, Scopus and Google Scholar

- Research which is freely available for redistribution

Submit your manuscript at www.biomedcentral.com/submit
C Biomed Central 